\title{
Milling Dynamics and Propagation of Mechanically Activated Self-Sustaining Reactions
}

\author{
Alberto Cincotti $\mathbb{D}^{D}$, Gabriele Traversari $\mathbb{D}^{\mathbb{D}}$, Giorgio Pia ${ }^{(D)}$, and Francesco Delogu \\ Dipartimento di Ingegneria Meccanica Chimica e dei Materiali, Università degli Studi di Cagliari, via Marengo 2, \\ Cagliari 09123, Italy \\ Correspondence should be addressed to Giorgio Pia; giorgio.pia@dimcm.unica.it
}

Received 23 July 2020; Revised 24 September 2020; Accepted 9 October 2020; Published 28 October 2020

Academic Editor: Hamed Akhavan

Copyright (c) 2020 Alberto Cincotti et al. This is an open access article distributed under the Creative Commons Attribution License, which permits unrestricted use, distribution, and reproduction in any medium, provided the original work is properly cited.

\begin{abstract}
This work focuses on the propagation of mechanically activated self-sustaining reactions during the mechanical processing of powder in ball mills. We use a numerical model to reconstruct the dynamics of a single ball and powder particles inside the reactor of a SPEX Mixer/Mill 8000 under operational conditions. Taking advantage of the analytical description of the reactor swing, the equations of motion of ball and powder particles are solved numerically. The discrete element method is used to describe contacts. Reaction is ignited in an individual particle randomly selected among those compressed during an impact between ball and reactor. A simple kinetic law and a set of rules involving degree of chemical conversion and distance between particles are used to obtain a phenomenological description of the reaction propagation. We show that the propagation is significantly affected by reaction rate in individual particles, with other factors being less influential. We observe a strong coupling between the dynamics of powder particles and the reaction propagation.
\end{abstract}

\section{Introduction}

Mechanical processing by ball milling (BM) is a powder metallurgy methodology that uses collisions between milling tools to induce physical and chemical changes in granular bodies confined inside a reactor $[1,2]$. When the milling tools collide, their surfaces trap a small fraction of the powder charge, thus subjecting it to mechanical loading $[3,4]$. Nonhydrostatic mechanical stresses arise across the network of contacts between the powder particles, giving rise to a disordered network of mechanical forces acting on the level of individual particles [4]. Local particle configurations influence the way local stresses distribute. Thus, individual particles can experience loading conditions differing in nature, rate, and intensity. Correspondingly, the loaded material can exhibit different local physical and chemical responses. Frictional heat production, cold-welding, fracturing, plastic deformation, and work-hardening processes that take place on the macroscopic scale $[1,3,5]$ can be accompanied, on the microscopic one, by the forced mixing of chemical species and the activation of physical and chemical transformations [6-16].

Although such transformations typically have gradual kinetics, often extending over long processing times $[1,2,17-19]$, highly exothermic systems can undergo selfsustaining high-temperature reactions $[1,2,17,20]$. These are characterized by a two-stage kinetics, with the initial microstructural refinement promoted by BM suddenly replaced by a chemical transformation able to proceed autonomously without any further input of mechanical energy due to the fast liberation of the reaction heat [20]. Ignition marks the discontinuity between the two stages and makes mechanically activated self-sustaining high-temperature reactions (MSRs) a special case of self-propagating high-temperature synthesis [21-25].

Ignition represents a crucial aspect of MSRs and, thus, significant attention has been paid to its study [20,26-35]. In particular, systematic experimental investigation aimed at measuring the activation time preceding the ignition of the MSR, commonly referred to as the ignition time, which has 
been shown to depend on several factors including chemical composition, impact energy, amount of reactants, and temperature [20, 26-35].

In contrast, much less attention has been devoted to the mechanisms governing the MSR propagation inside the mechanochemical reactor under operational conditions. In principle, it is quite reasonable to expect that the dynamics of milling tools and powder particles can affect the degree of chemical conversion and the nature of final products. Nevertheless, the direct experimental investigation of the propagation process raises considerable difficulties due to the rapid motion of the reactor, the violent stirring of the powder charge, the scarcely predictable ignition time, and the high rate of chemical conversion. Overall, such factors translate into fragmentary and contradictory empirical evidence simply suggesting that MRSs do not result necessarily in the complete conversion of reactants to products and that the variation of $\mathrm{BM}$ conditions can even determine the suppression of MRSs [20, 26-35]. Nevertheless, detailed information on the way milling dynamics can affect MSRs is highly desirable in view of a reliable exploitation of MRSs in materials manufacturing contexts.

Based on the considerations above, the present study aims at throwing light on the way MSRs propagate across the powder charge under operational conditions. To this aim, we use numerical simulations to investigate the dynamics of a granular body inside the reactor of a SPEX Mixer/Mill 8000 ball mill in the presence of a single milling ball. The reactor swing gives rise to a granular flow with internal dynamics determined by the milling frequency. Ignition is simply modelled by activating the chemical conversion in a single powder particle and describing the reaction propagation to other particles via a phenomenological set of rules. We show that different propagation modes can exist depending on the milling frequency and the propagation rate.

Details of numerical methods are given in the following.

1.1. Numerical Methods. Numerical simulations take advantage of the mathematical description of the reactor motion in a SPEX Mixer/Mill 8000. Manufactured by SPEX SamplePrep, LLC, the ball mill is equipped with a $230 \mathrm{~V}-50 \mathrm{~Hz}$ motor that rotates at $1425 \mathrm{rpm}$ and activates an eccentric fulcrum through a pulley system. The fulcrum is rigidly connected with a mechanical arm that has a clamp assembly to secure the reactor at the end. A recall spring moderates the arm swing. The reactor consists of a cylindrical container with inner height and diameter equal to about 5.8 and $3.8 \mathrm{~cm}$, respectively. Once the reactor is clamped to the mechanical arm, its geometrical centre lies on the axis of the mechanical arm at about $10 \mathrm{~cm}$ from the fulcrum axis. The reactor undergoes an angular harmonic displacement on the vertical plane and a synchronous rotation around the axis of the mechanical arm at a frequency. The angular swings on the vertical horizontal planes have amplitudes, respectively, $\theta_{0}$ and $\alpha_{0}$, equal to $15^{\circ}$. Full details can be found elsewhere $[36,37]$.

An inertial Cartesian reference frame centred on the eccentric fulcrum can be used to describe the trajectory of any point of the reactor $[36,37]$. The dynamics of balls and powder particle inside the reactor can be conveniently described with reference to a noninertial Cartesian frame moving with the reactor and with the origin at its centre $[36,37]$. The inertial and noninertial Cartesian reference frames are shown in Figure 1 for illustrative purpose. Since acceleration components along inertial Cartesian axes take values about 20 times larger than gravity, we neglect them.

Calculations were performed considering a cylindrical reactor approximately $5.8 \mathrm{~cm}$ in height and $1.9 \mathrm{~cm}$ in radius. The reactor contained 5000 spherical particles with mass of $0.7 \mathrm{mg}$ and diameter of $0.3 \mathrm{~mm}$. A single milling ball with mass and diameter equal to $8 \mathrm{~g}$ and $1.24 \mathrm{~cm}$, respectively, was considered. Powder particles and milling ball occupy, approximately, $0.11 \%$ and $1.52 \%$ of the reactor volume, respectively. The ball-to-powder mass ratio is equal to about 2.28. This value is well within the range of ball-to-powder mass ratios typically utilized in milling experiments, which can usually be as small as 0.5 and as large as $24[1,2,17]$.

In the absence of contacts with each other or with the reactor wall and bases, ball and powder particles undergo uniform rectilinear motion. Contacts between balls, powder particles, and reactor walls are described using the discrete element method $[38,39]$. Accordingly, the effects of normal and tangential forces on the colliding bodies are evaluated using a combination of springs and dashpots [38, 39]. Since accurate calculations indicate that tangential forces typically are much smaller than normal ones, in this study, they have been neglected. Normal forces are expressed as

$$
N=-\kappa \varepsilon-\gamma \frac{\mathrm{d} \varepsilon}{\mathrm{d} t} .
$$

This equation describes the force of a damped harmonic oscillator with repulsion and dissipation terms that allow modelling partially elastic collisions. Thus, $\kappa$ is Hooke's constant, $\gamma$ is a damping coefficient, $\varepsilon$ is the deformation undergone by the colliding surfaces, and $t$ is time $[38,39]$. Force parameters are $4.14 \times 10^{6} \mathrm{~kg} \cdot \mathrm{s}^{-2}$ and $5.5452 \times 10^{1} \mathrm{~kg} \cdot \mathrm{s}^{-1}$ for collisions with the reactor wall and $3.622 \times 10^{2} \mathrm{~kg} \cdot \mathrm{s}^{-2}$ and $4.85 \times 10^{-3} \mathrm{~kg} \cdot \mathrm{s}^{-1}$ for collisions among particles and milling ball. Equations of motion were integrated using the Verlet algorithm with a time step of $5 \mu \mathrm{s}[39,40]$. The milling frequency, $\nu$, was varied in the interval between 11.6 and $21.6 \mathrm{~Hz}$.

For convenience, the initial configuration of powder particles and ball consisted of an ordered array not in contact with reactor wall and bases. Order is lost very quickly once ball and powder particles collide with each other and with the reactor wall and bases. Ball and powder particle dynamics have fully relaxed after two complete reactor swings and the resulting disordered dynamics were no longer affected by initial conditions.

Once the dynamics have suitably relaxed, a powder particle randomly selected among those compressed by the ball against one of the reactor bases during an impact is ignited. The reaction in individual particles is given a phenomenological description using the Johnson-MehlAvrami kinetics [39]. Accordingly, the conversion degree is related to time by the expression 


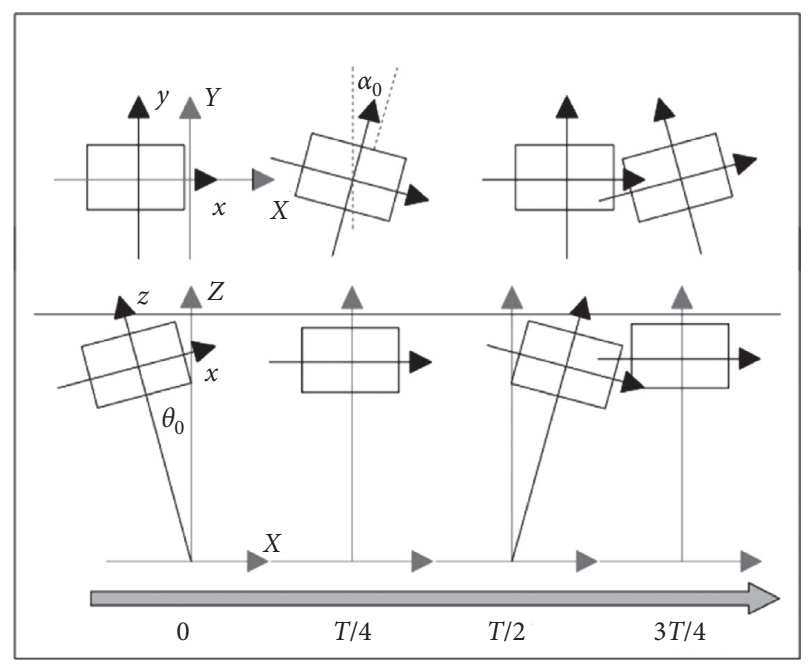

FIGURE 1: Inertial and noninertial Cartesian reference frames used to describe the reactor swing and the dynamics of ball and powder particles inside the reactor.

$$
\alpha=1-\exp \left(-k t^{n}\right)
$$

where $k$ is the rate constant, $t$ is time, and $n$ is the Avrami exponent. Based on available literature [41], $n$ was given the value of 1.649 .

Reaction propagation from a given particle to a neighbouring one is modelled by two simple phenomenological rules that must be satisfied simultaneously. First, a nonreacting particle can be ignited by a reacting one if it is distant from the latter less than a selected distance, $d_{\mathrm{ig}}$. Second, a reacting particle can ignite a nonreacting particle only if its conversion degree $\alpha$ is between two suitably chosen set values $\alpha_{\min }$ and $\alpha_{\max }$. For convenience, $\alpha_{\max }$ was set equal to $1-\alpha_{\min }$.

These rules undeniably provide only an oversimplified description of the reaction propagation. At the same time, however, they make the simulation of the ignition and propagation processes affordable. In this regard, it is worth noting that a rigorous description of the mechanically activated ignition, and of the subsequent propagation, is not only out of the scope of the present work but also, presently, out of reach of routine scientific computation. The physics and chemistry involved render the problem highly complex. On the one hand, simulations have to properly describe the mechanics of impacts and the associated dissipation of mechanical energy of ball and powder particles. On the other hand, they have to account for the thermodynamics and kinetics of a rapid chemical transformation taking place out of equilibrium in an ever-changing environment. The chemical transformation involves individual particles, which are also the primary heat sources due to the liberation of the latent heat of reaction. Any given particle is a world of its own that exchanges heat with a turbulent gaseous atmosphere and, during impacts, with other particles by direct contact.

It is not easy to combine the discrete dynamics of individual particles with the continuum of atmosphere while describing the underlying heat transfer processes. However, it is not difficult to identify the most important factors determining the ignition and the propagation of the chemical reaction. First, the higher the chemical transformation rate, the higher the rate of temperature increase in individual particles, which results in a more efficient heat transfer. Second, the shorter the distance between two particles, the more intense and the easier the heat exchange between them.

It is precisely from these two considerations that the phenomenological rules used in simulations are derived. The choice of a minimum distance to allow ignition reminds of the description of an effective heat transport inside a granular flow. The choice of a conversion degree interval relates to the description of the heat generation rate. This latter typically involves a maximum, being limited by a low temperature initially and by the progressive depletion of reactants subsequently. Using these two criteria allows avoiding the rigorous approach based on the combination of heat generation rate inside each particle with the thermal conduction and radiation between particles, reactor walls, and milling ball. Simultaneously, it allows capturing the fundamental features of the heat transfer processes, making simulations feasible.

Simulations were performed varying the frequency of the reactor swing, $v$, the reaction constant, $k$, the ignition distance, $d_{\mathrm{ig}}$, and the lower threshold of conversion degree, $\alpha_{\text {min }}$. Parameter values were combined as summarized in Table 1 to explore the effect of BM conditions on the reaction propagation. In particular, the reactor swing frequency $\nu$ was varied between 11.6 and $21.6 \mathrm{~Hz}$, the rate constant $k$ between $6.845 \times 10^{-2}$ and $6.845 \times 10^{2} \mathrm{~s}^{-1}$, and the ignition distance $d_{\mathrm{ig}}$ between $7 \times 10^{-5}$ and $7 \times 10^{-4} \mathrm{~m}$. Similarly, $\alpha_{\min }$ was varied between 0.05 and 0.30 .

\section{Results and Discussion}

The initial configuration used to start simulations has been suitably chosen to make relaxation as faster as possible. The positions of ball and powder particles inside the reactor are shown in Figure 2(a). The reactor is at the inversion point of its swing. While ball and powder particles are at rest, the reactor base accelerates toward ball and powder particles. The first contacts between the reactor base and cylindrical wall, on the one hand, and the ball and powder particles, on the other hand, trigger the disordered dynamics of the granular system. This is entirely involved within a quarter of the reactor swing period. Information about starting conditions is lost within two cycles of the reactor swing, almost independent of the milling frequency. Once relaxed, the system reaches steady-state dynamics characterized by the displacement of ball and powder particles between the opposite reactor bases.

The simple visualization of the system evolution in time allows capturing the crucial features of granular flow (see Supplementary Information-video_propagation.avi available here). A suitably chosen system configuration is shown in Figure 2(b) for illustrative purposes. It can be seen that powder particles do not scatter throughout all the available 
TABLE 1: Parameter values utilized to carry out numerical simulations. Data refer to variable parameters, namely, the frequency $v(\mathrm{~Hz})$ of the reactor swing, the rate constant $k\left(\mathrm{~s}^{-1}\right)$ of the Johnson-Mehl-Avrami kinetic law, the ignition distance $d_{\mathrm{ig}}(\mathrm{m})$, and the lower ignition threshold of conversion degree $\alpha_{\min }$.

\begin{tabular}{|c|c|c|c|c|}
\hline Simulation run & $v$ & $k$ & $d_{\mathrm{ig}}$ & $\alpha_{\min }$ \\
\hline 1 & 14.6 & $6.845 \times 10^{2}$ & $7 \times 10^{-4}$ & 0.3 \\
\hline 2 & 21.6 & $6.845 \times 10^{2}$ & $7 \times 10^{-4}$ & 0.3 \\
\hline 3 & 21.6 & $6.845 \times 10^{2}$ & $7 \times 10^{-5}$ & 0.3 \\
\hline 4 & 14.6 & $6.845 \times 10^{2}$ & $7 \times 10^{-5}$ & 0.3 \\
\hline 5 & 14.6 & $6.845 \times 10^{1}$ & $7 \times 10^{-4}$ & 0.3 \\
\hline 6 & 14.6 & $6.845 \times 10^{1}$ & $7 \times 10^{-5}$ & 0.3 \\
\hline 7 & 21.6 & $6.845 \times 10^{1}$ & $7 \times 10^{-4}$ & 0.3 \\
\hline 8 & 21.6 & $6.845 \times 10^{1}$ & $7 \times 10^{-5}$ & 0.3 \\
\hline 9 & 14.6 & $6.845 \times 10^{0}$ & $7 \times 10^{-4}$ & 0.3 \\
\hline 10 & 14.6 & $6.845 \times 10^{0}$ & $7 \times 10^{-5}$ & 0.3 \\
\hline 11 & 21.6 & $6.845 \times 10^{0}$ & $7 \times 10^{-4}$ & 0.3 \\
\hline 12 & 21.6 & $6.845 \times 10^{0}$ & $7 \times 10^{-5}$ & 0.3 \\
\hline 13 & 14.6 & $6.845 \times 10^{-1}$ & $7 \times 10^{-5}$ & 0.3 \\
\hline 14 & 14.6 & $6.845 \times 10^{-2}$ & $7 \times 10^{-5}$ & 0.3 \\
\hline 15 & 14.6 & $6.845 \times 10^{-2}$ & $7 \times 10^{-5}$ & 0.05 \\
\hline 16 & 11.6 & $6.845 \times 10^{2}$ & $7 \times 10^{-4}$ & 0.3 \\
\hline
\end{tabular}

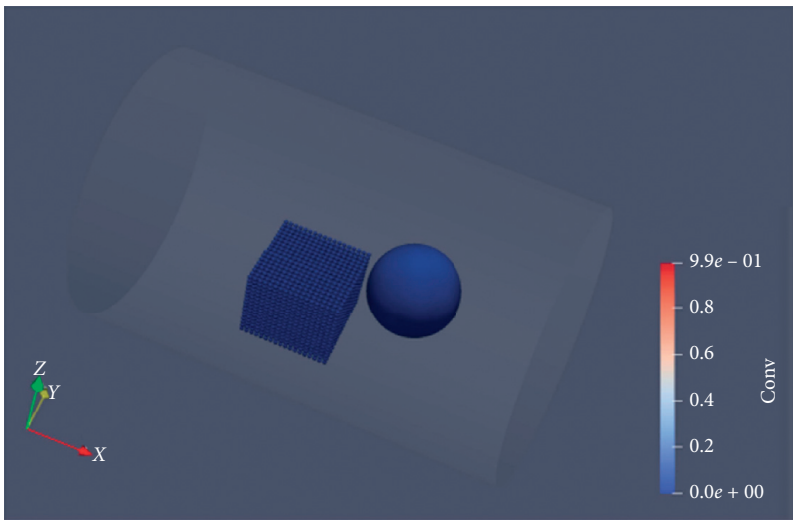

(a)

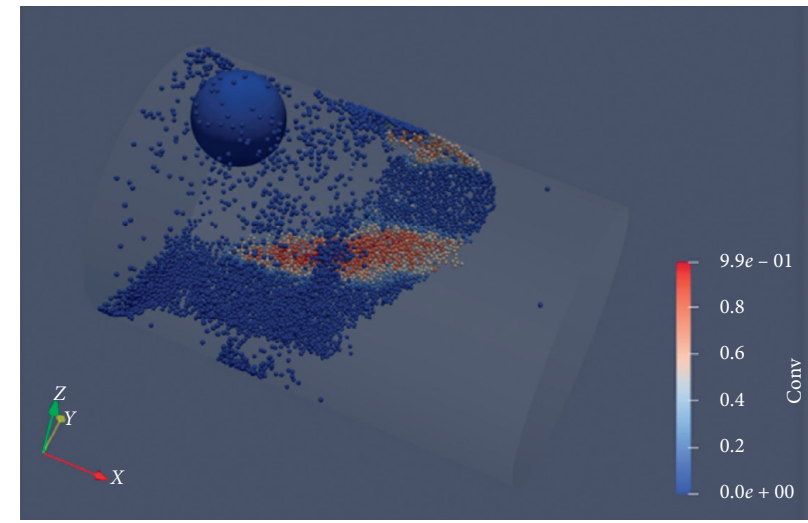

(b)

Figure 2: (a) Initial and (b) relaxed configurations of ball and powder particles. Data refer to the case of a reactor swung at $14.6 \mathrm{~Hz}$. Inside the reactor, an MSR is propagating. The colour of individual powder particles measures the degree of chemical conversion (blue-low conversion degrees; red-high conversion degrees).

reactor volume but rather occupy a relatively small portion of it. The continuous energy exchange within the granular flow and between powder particles and milling tools makes the dynamics strongly dissipative. Accordingly, it never results in particle configurations spreading across the entire reactor volume. Although individual powder particles undergo intrinsically disordered dynamics, the granular body remains quite compact in its whole. Accelerations due to the synchronous reactor rotations and oscillations tend to push the powder particles against the reactor walls. At any time, only a few powder particles can be found to be moving freely across the reactor volume along trajectories diverging from the mass of the granular body, mostly caused by energetic rebounds within the granular flow. The vast majority of powder particles simply remain in contact with the reactor walls or quite close to them.

Consequent to the dissipative dynamics experienced by the powder particles, the granular flow couples its dynamics with the reactor movement. Accordingly, the displacement of the granular body in its whole between the opposite reactor bases is definitely periodic. This clearly emerges from the numerical analysis of the granular flow with reference to the noninertial Cartesian frame, which has the $x$-axis coincident with the main axis of the cylindrical reactor. The quantity that allows a suitable insight into the dynamics of individual powder particles is their $x$ coordinate. Its variation in time provides a reliable description of the main displacements undergone by each particle. In turn, the statistical distribution of the $x$ coordinate of powder particles gives a relatively clear idea of the movement undergone by the granular body.

The statistical distributions of the $x$ coordinate obtained at different points along the reactor cycle are shown in Figure 3. Distribution curves are quite different from each other and suggest the occurrence of contractions and expansions of the granular body in response to the mechanical 


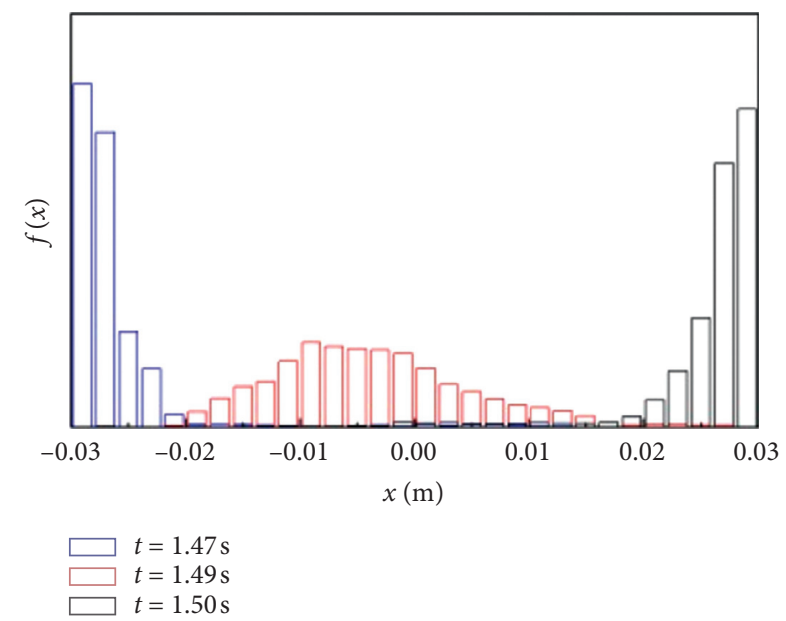

Figure 3: Distributions $f(x)$ of the $x$ coordinates of powder particles. Data refer to three different times during a simulation run investigating the ball and powder particle dynamics inside a reactor swung at $14.6 \mathrm{~Hz}$. The bin size is equal to $0.002 \mathrm{~m}$.

forces acting during the reactor cycle. At the lowest time considered, the reactor has just passed the point of maximum velocity in the inertial Cartesian frame. As it decelerates, powder particles start detaching from the reactor base and begin traveling across the reactor from one base to the other. Correspondingly, the granular body undergoes the first expansion stages along the main axis of the cylindrical reactor. While powder particles move toward the opposite base, mostly close to the cylindrical wall, the reactor keeps decelerating. At the intermediate time considered, the reactor is between the point of maximum velocity and the inversion point of its motion. The granular body has fully expanded under the effect of accelerations due to oscillations and rotations and the corresponding distribution curve of $x$ coordinate appears quite broad. Shortly after the reactor has started moving in the opposite direction, the first powder particles come into contact with the reactor base and the granular body progressively undergoes a contraction. Reactor and granular body have opposite movement and the powder particles regroup on the reactor base. A new expansion stage of the granular body takes place when the reactor reaches the point of maximum velocity. The cycle is repeated and the granular flow exhibits periodicity synchronous to the reactor swing.

The ball has a disordering effect on the dissipative dynamics of the granular body. Due to its significantly larger mass and size, it easily pushes away any powder particle that comes into contact with it. Therefore, at least on the average, contacts between ball and granular body result in the scattering of powder particles along different directions. Vice versa, powder particles have an ordering effect on the ball dynamics. At any given close approach between ball and reactor wall and bases, a certain number of powder particles are trapped. Compressed between the surfaces of ball and reactor, they absorb part of the mechanical energy of the impact, thus softening the collision elasticity. A certain amount of the ball energy at impact is dissipated in the deformation work of powder particles and another amount is spent in the scattering of powder particles away from the impact point. It follows that the ball undergoes partially elastic impacts that can be approximately described by a restitution coefficient of about 0.5 . On the average, the ball undergoes two main impacts with the reactor bases per cycle of the reactor swing. Therefore, ball impact frequency is twice the frequency of the reactor swing, in agreement with previous studies [36, 37].

Since the compression of powder particles and the consequent deformation processes are accompanied by local temperature rises $[1,2,17,20]$, the MSR was initiated during impacts. In particular, one of the powder particles undergoing compression was randomly selected and its chemical conversion was allowed to change, from that moment, according to equation (2).

Once ignited, the reaction proceeds in individual particles at a rate determined by the selected Johnson-MehlAvrami kinetics. For the sake of illustration, let us consider three powder particles close to each other in the initial ordered configuration. These powder particles ignite at different times during the simulation run with the first parameters listed in Table 1. Their degrees of chemical conversion, $\alpha$, are shown in Figure 4(a) as a function of time, $t$. First, it can be seen that the reaction takes place on a time scale comparable with the period of the reactor swing, reaching completion in about $6 \times 10^{-2} \mathrm{~s}$. Second, although the powder particles were initially nearest neighbours, their ignition times are different. This is a clear result of the effective disordering dynamics governing ball and granular body inside the moving reactor. Whereas the reaction rate in individual particles is determined exclusively by the Johnson-Mehl-Avrami kinetics and is, thus, independent of external factors, reaction propagation from one particle to the other can be expected to depend on the dynamics of the granular body. In this regard, it is useful to refer to the cumulative chemical conversion degree.

$$
\alpha_{\text {cum }}(t)=\frac{1}{N_{p}} \sum_{i=1}^{N_{i g}(t)} \alpha_{i}(t),
$$

which represents the overall conversion degree averaged over the total number of powder particles, $N_{p}$. In particular, the cumulative conversion degree $\alpha_{\text {cum }}(t)$ at time $t$ is obtained by summing the conversion degrees $\alpha_{i}(t)$ at time $t$ of every reacting particle. Of course, the number of reacting particles, $N_{i g}(t)$, varies with time $t$. It follows that $\alpha_{\text {cum }}(t)$ equals 1 only when all of the $N_{p}$ powder particles forming the granular body have reacted completely.

The cumulative conversion degree $\alpha_{\text {cum }}(t)$ for the simulation run with the first parameters listed in Table 1 is shown in Figure 4(b) as a function of time $t$. The curve exhibits sigmoidal shape. Although apparently smooth, it presents several slope variations that appear as small irregularities and shoulders. This clearly suggests that the reaction does not propagate steadily across the granular body. Rather, we can expect that it is affected by the granular flow dynamics. 


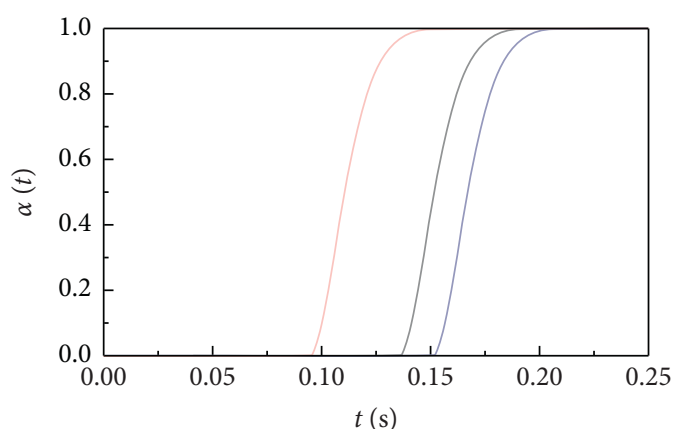

(a)

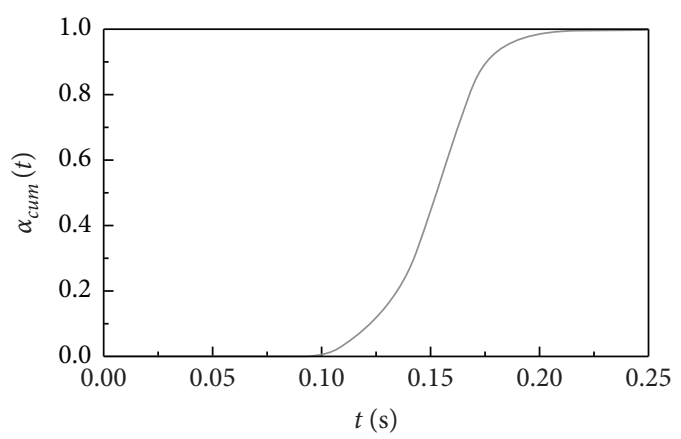

(b)

Figure 4: (a) The degree of chemical conversion, $\alpha(t)$, for three particles that are nearest neighbours in the initial configuration and (b) the cumulative degree of chemical conversion, $\alpha_{\text {cum }}(t)$, as a function of time, $t$. Data refer to the first simulation described in Table 1.

The irregular progress of the MSR can be suitably pointed out taking into due account the ignition time, $t_{i g}$, of individual powder particles. Ignition times, indeed, reveal when individual particles get involved in the reaction. The cumulative distribution of $t_{i g}$ over the entire set of powder particles is shown in Figure 5. Their first derivatives are also shown to highlight irregularities. Data refer to four representative case studies that differ from each other for the value of the rate constant $k$ of the Johnson-Mehl-Avrami reaction kinetics. Specifically, we reduced the $k$ value from $6.845 \times 10^{2}$ to $6.845 \times 10^{-1} \mathrm{~s}^{-1}$, with the other parameters being the same.

According to general expectations, reaction propagation undergoes a corresponding deceleration. In all the cases, cumulative distributions clearly reveal irregular propagation processes characterized by sudden rises in the number of reacting particles followed by shoulders and even plateaus extending over time intervals of variable length. The first derivative of cumulative distributions shows that the number of reacting particles changes discontinuously. In particular, data show that a significant number of new particles are ignited in correspondence of the inversion points of the reactor swing, marked by the vertical-dotted lines in Figure 5, or close to them. Accordingly, we can readily infer that ignition of individual particles takes place in bursts, mostly related to the contractions that the granular body undergoes periodically as a result of the reactor swing. It is during contraction stages that powder particles come into closer contact, thus fulfilling the ignition condition governed by ignition distance $d_{\mathrm{ig}}$. The comparison between plots in Figure 5 indicates that the smaller the rate constant $k$, the more evident the above-mentioned behaviour. The first derivative of the cumulative distribution of ignition times for the simulation run with the smaller $k$ value exhibits distinct peaks of propagation in correspondence of the contractions of granular flow caused by the impingement of powder particles on the reactor base. Further reduction of the $k$ value can be expected to make the effect even more evident on longer simulation times.

The variation of simulation parameters affects reaction propagation and, then, cumulative conversion degree $\alpha_{\text {cum }}(t)$ as shown in Figure 6. Most evident effects stem from the variation of the rate constant $k$ accounting for the reaction rate in individual powder particles. Data in Figure 6(a) show that reaction propagation significantly accelerates as $k$ increases. This can be ascribed to the rapid attainment in reacting powder particles of conversion degrees higher than the minimum threshold $\alpha_{\min }$ required to activate the reaction in nonreacting powder particles distant less than $\mathrm{d}_{i g}$. Once a certain number of powder particles start reacting, reaction propagation to nonreacting particles during contractions of the granular body can be very fast. In most favourable cases, even a single contraction can be sufficient to obtain complete conversion within a single reactor swing.

The frequencies of the reactor swing, $v$, and the ignition distance, $d_{\mathrm{ig}}$, are much less influential than the minimum threshold for individual particle ignition, $\alpha_{\min }$. It can be seen from Figures $6(\mathrm{~b})$ and $6(\mathrm{c})$ that reaction propagation accelerates only slightly as $v$ and $d_{\text {ig }}$ increase. In the former case, acceleration is ascribable to the direct relationship existing between the frequency of the reactor swing and the frequency of contractions in the granular flow. Contractions favour the reaction propagation across the granular body as a consequence of the reduced distances between loose powder particles. Thus, any $v$ increase determines a corresponding increase of the reaction propagation once a sufficient number of particles have conversion degree higher than $\alpha_{\text {min }}$. Similarly, an increase of the minimum distance required for a reacting particle to activate the reaction in a neighbouring, nonreacting particle favours reaction propagation. In particular, the longer $d_{\mathrm{ig}}$, the higher the probability of propagating the MSR in the granular body when its contraction is not maximum.

Numerical findings indicate that the propagation of MSRs in a SPEX Mixer/Mill 8000 ball mill depends on factors related to the reactor swing and the intrinsic reaction rate in individual powder particles. The numerical model takes advantage of the accurate description of the ball and powder particle dynamics inside the moving reactor, based, in turn, on a reliable reconstruction of contact dynamics. Numerical simulations clearly show that powder particles form a granular body governed by strongly dissipative dynamics. The fast energy redistribution among powder particles allows the granular flow to exhibit a periodic behaviour. Despite the disordered trajectories of individual powder particles, the granular 


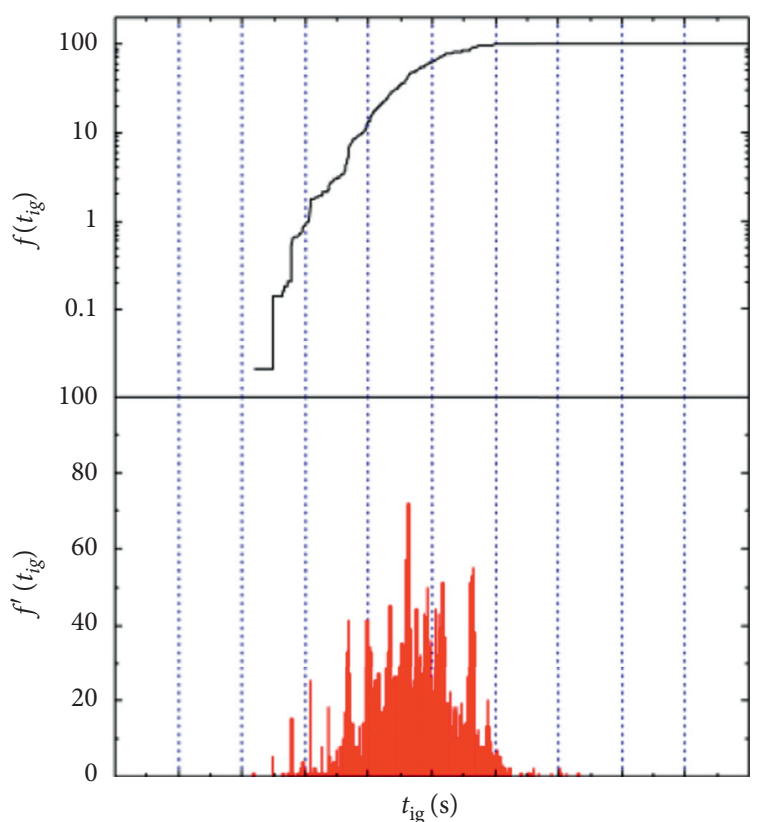

(a)

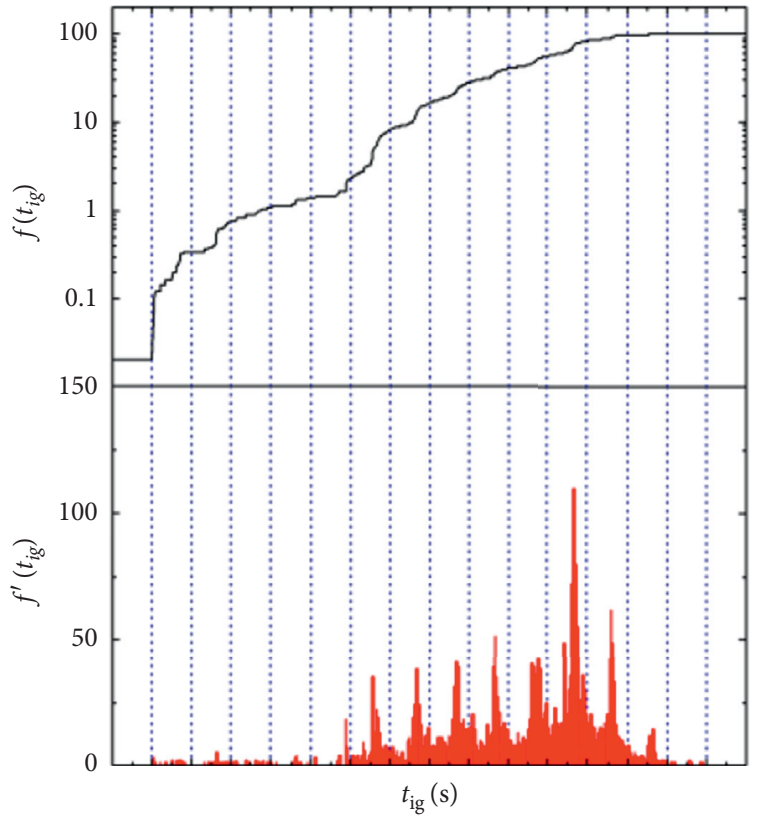

(c)

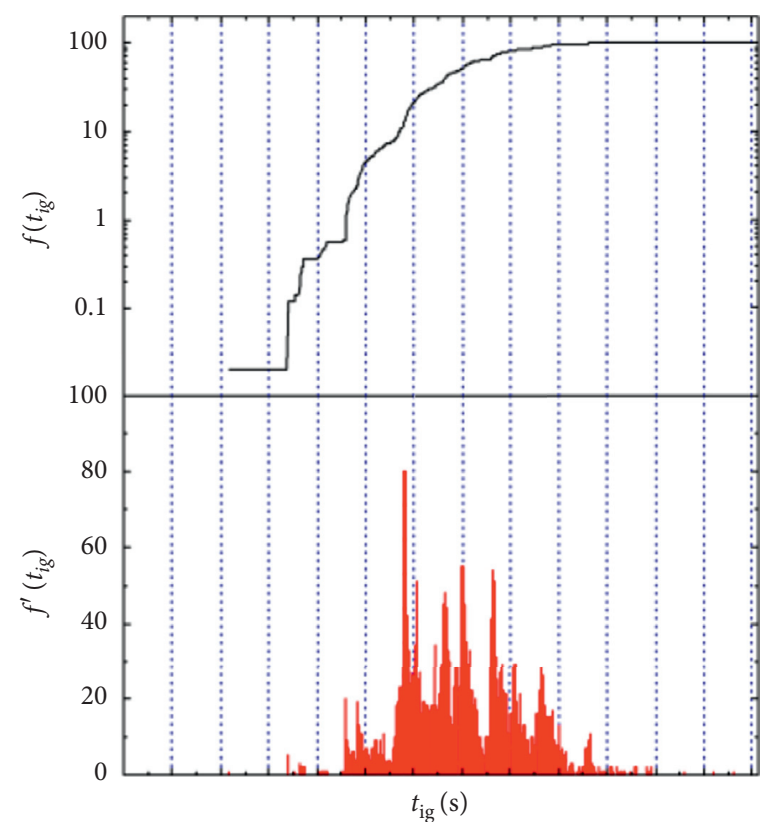

(b)

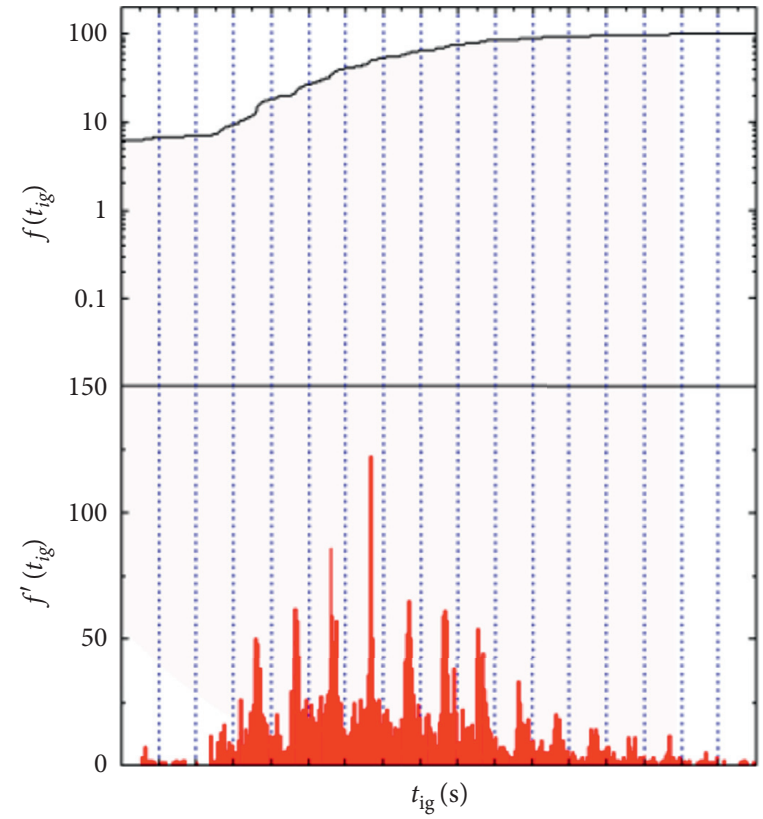

(d)

Figure 5: Cumulative distributions of ignition times (upper panel) and their first derivative (lower panel) over the whole set of powder particles for the fourth (a), sixth (b), tenth (c), and thirteenth (d) simulations described in Table 1. Vertical-dashed lines correspond to the inversion points of the reactor swing. The bin size varies from $3.5 \times 10^{-4}$ to $1.4 \times 10^{-3} \mathrm{~s}$.

body undergoes quite regular displacements between the two opposite reactor bases. Overall, the granular flow is characterized by alternating contraction and expansion stages. Contractions take place when the granular body moving in a certain direction impacts the reactor base moving in the opposite direction, whereas expansion starts when the reactor has reached the point of maximum velocity. Two contraction and two expansion stages occur per cycle of the reactor swing.
The granular flow is disordered by the ball. Much bigger than powder particles, the ball is able to push them away. Accordingly, the powder particles are scattered across the reactor volume. Every time the ball impacts the reactor wall or bases, it compresses a relatively small number of powder particles. This results in a significant dissipation of the ball mechanical energy during the impact, which allows the establishment of a partially inelastic impact regime. As a consequence, the ball undergoes two main impacts per cycle 


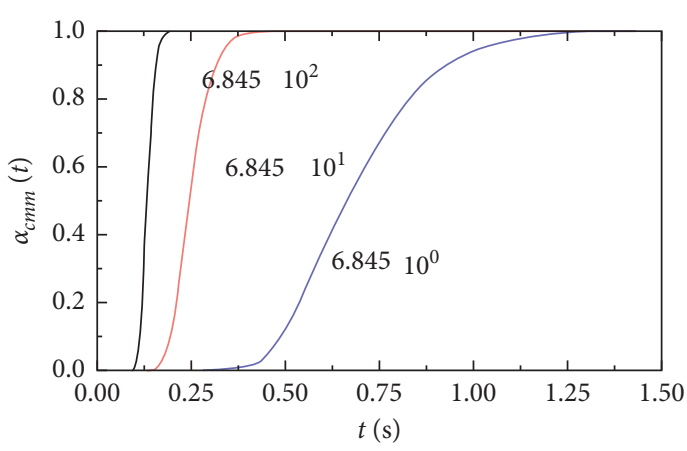

(a)

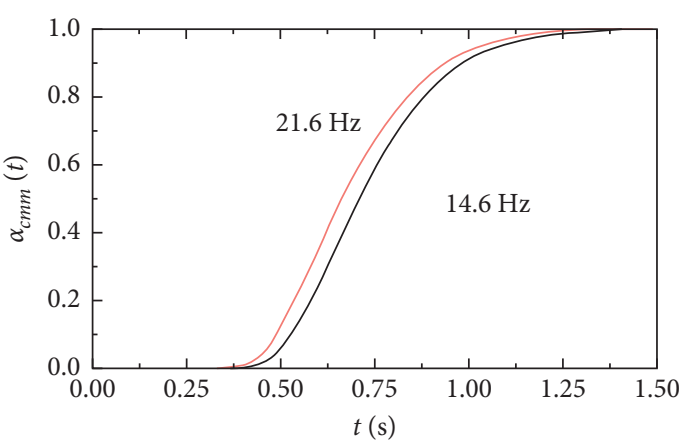

(b)

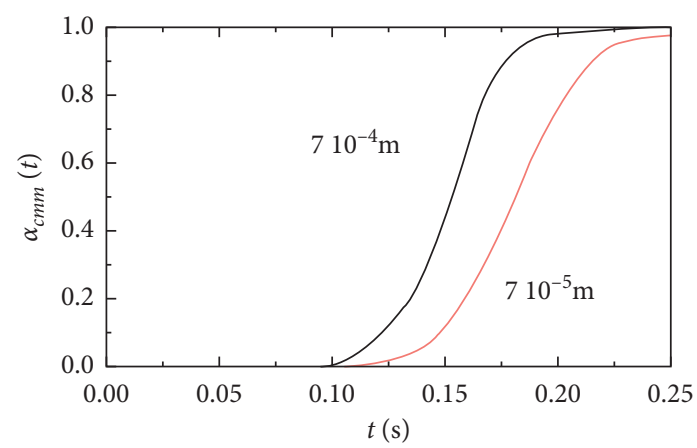

(c)

Figure 6: The cumulative degree of chemical conversion, $\alpha_{\text {cum }}(t)$, as a function of time, $t$, obtained from simulation runs with different values of (a) rate constant of the Johnson-Mehl-Avrami kinetics, (b) frequency of the reactor swing, and (c) ignition distance. Parameter values are shown.

of the reactor swing within the whole range of reactor swing frequency explored. Thus, the results obtained satisfactorily agree with experimental and numerical evidence from previous studies.

We have used the numerical reconstruction of ball and powder particles trajectories to investigate the relationship between the granular flow dynamics and the propagation of MSRs inside a moving reactor. To this aim, we have chosen to describe the reaction of a single powder particle according to a Johnson-Mehl-Avrami kinetics. Although phenomenological, this description is sufficient to enable the evaluation of factors potentially affecting the MSR propagation. Reaction was first ignited in a single particle randomly chosen among those compressed by the ball during an impact with the reactor base. We observe that the propagation strongly depends on the rate constant of the phenomenological kinetic law. In particular, propagation is favoured by large values of the rate constant. Less influential factors are the distance at which a reacting particle can ignite the MSR in a nonreacting particle and the frequency of the reactor swing. In any case, numerical findings clearly show that the MSR proceeds via an irregular, discontinuous propagation process. Specifically, bursts in the reaction ignition in individual powder particles occur approximately in correspondence of the contractions undergone by the granular flow as a result of the intrinsic dynamics of the granular body.

Our results allow deeper insight into the dynamics governing the motion of ball and powder particles inside a moving reactor of the SPEX Mixer/Mill 8000 and helps in clarifying the conditions under which MSRs propagate. Further work is needed to perform a systematic exploration of the parameter space.

\section{Data Availability}

The data used to support the findings of this study are included within the article.

\section{Disclosure}

Gabriele Traversari performed his activity within the framework of the International Ph.D. in Innovation Sciences and Technologies at Università degli Studi di Cagliari, Italy.

\section{Conflicts of Interest}

The authors declare that there are no conflicts of interest.

\section{Acknowledgments}

The authors express their gratitude to Professor Laszlo Takacs for useful discussions and the critical reading of the manuscript. This work is dedicated to his memory. Financial support has been given by the University of Cagliari (Grant no. RICCAR_2012_Delogu). 


\section{Supplementary Materials}

System evolution in time of granular flow in the noninertial Cartesian frame moving with the reactor: trajectories of 5000 particles and a milling ball. The colour of individual powder particles measures the degree of chemical conversion (blue, low conversion degrees; red, high conversion degrees). Data refer to the case of a reactor swung at $14.6 \mathrm{~Hz}$ (i.e., RUN 1 in Table 1). (Supplementary Materials)

\section{References}

[1] M. Sopicka-Lizer, Ed., Mechanochemical Processing of Nanopowders, Woodhead Publishing, Cambridge, UK, 2010.

[2] F. Delogu and G. Mulas, Eds., Experimental and Theoretical Studies in Modern Mechanochemistry, Transworld Research Network, Kerala, India, 2010.

[3] T. H. Courtney, "Process modeling of mechanical alloying," Materials Transaction JIM, vol. 36, pp. 110-122, 1995.

[4] F. Delogu, "Are processing conditions similar in ball milling and high-pressure torsion? The case of the tetragonal-tomonoclinic phase transition in $\mathrm{ZrO}_{2}$ powders," Scripta $\mathrm{Ma}$ terial, vol. 67, no. 4, pp. 340-343, 2012.

[5] D. R. Maurice and T. H. Courtney, "The physics of mechanical alloying: a first report," Metallurgical Transaction A, vol. 21, pp. 289-303, 1990.

[6] X. Y. Fu, M. L. Falk, and D. A. Rigney, "Sliding behavior of metallic glass: Part II. Computer simulations," Wear, vol. 250, no. 1, pp. 420-430, 2001.

[7] K. Kadau, T. C. Germann, P. S. Lomdahl, and B. L. Holian, "Microscopic view of structural phase transitions induced by shock waves," Science, vol. 296, pp. 1681-1684, 2002.

[8] A. C. Lund and C. A. Schuh, "Atomistic simulation of straininduced amorphization," Applied Physics Letters, vol. 82, no. 13, pp. 2017-2019, 2003.

[9] S. Odunuga, Y. Li, P. Krasnochtchekov, P. Bellon, and R. S. Averback, "Forced chemical mixing in alloys driven by plastic deformation," Physical Review Letter, vol. 95, no. 4, pp. 93-96, 2005.

[10] F. Delogu and G. Cocco, "Numerical simulations of atomicscale disordering processes at impact between two rough crystalline surfaces," Physical Review B, vol. 74, no. 3, Article ID 35406, 2006.

[11] F. Delogu, "Forced chemical mixing in model immiscible systems under plastic deformation," Journal of Applied Physics, vol. 104, no. 7, Article ID 073533, 2008.

[12] J. Song and D. J. Srolovitz, "Mechanism for material transfer in asperity contact," Journal of Applied Physics, vol. 104, no. 12, Article ID 124312, 2008.

[13] N. Q. Vo, S. Odunga, P. Bellon, and R. S. Averback, "Forced chemical mixing in immiscible alloys during severe plastic deformation at elevated temperatures," Acta Materialia, vol. 57, no. 10, pp. 3012-3019, 2009.

[14] F. Delogu, "Molecular dynamics of collisions between rough surfaces," Physical Review B: Condensed Matter and Materials Physics, vol. 82, no. 20, pp. 1-9, 2010.

[15] Y. Ashkenazy, N. Q. Vo, D. Schwen, R. S. Averback, and P. Bellon, "Shear induced chemical mixing in heterogeneous systems," Acta Materialia, vol. 60, no. 3, pp. 984-993, 2012.

[16] F. Delogu, "A possible alloying mechanism in idealized collisions between $\mathrm{Cu}$ and $\mathrm{Sn}$ crystals," Chemical Physics Letters, vol. 521, pp. 125-129, 2012.
[17] C. Suryanarayana, "Mechanical alloying and milling," Progress Material Science, vol. 46, no. 1-12, pp. 1-184, 2001.

[18] F. Delogu, "A combined experimental and numerical approach to the kinetics of mechanically induced phase transformations," Acta Materialia, vol. 56, no. 4, pp. 905-9012, 2008.

[19] J. S. Benjamin, "Fundamentals of mechanical alloying," Material Science Forum, vol. 88-90, pp. 1-18, 1992.

[20] L. Takacs, "Self-sustaining reactions induced by ball milling," Progress Material Science, vol. 47, no. 4, pp. 355-414, 2002.

[21] A. G. Merzhanov and I. P. Borovinskaya, "A new class of combustion processes," Combustion Science Technology, vol. 10, pp. 195-201, 1974.

[22] Z. A. Munir and U. Anselmi-Tamburini, "Self-propagating exothermic reactions: The synthesis of high-temperature materials by combustion," Mater Sci Reports, vol. 3, no. 7-8, pp. 277-365, 1989.

[23] Z. A. Munir and J. B. Holt, Combustion and Plasma Synthesis of High-Temperature Materials, VCH Publisher, Berlin, Germany, 1990.

[24] J. J. Moore and H. J. Feng, "Combustion synthesis of advanced materials: Part I. Reaction parameters," Progress Materials Science, vol. 39, no. 4-5, pp. 243-273, 1995.

[25] J. J. Moore and H. J. Feng, "Combustion synthesis of advanced materials .2. Classification, applications, and modeling," Progress Materials Science, vol. 39, no. 4-5, pp. 275-316, 1995.

[26] C. G. Tschakarov, G. G. Gospodinov, and Z. Bontschev, "Über den mechanismus der mechanochemischen Synthese anorganischer Verbindungen," Journal Solid State Chemistry, vol. 41, no. 3, pp. 244-252, 1982.

[27] V. Rusanov and C. Chakurov, "On the development mechanism and kinetics of the explosive mechanochemical synthesis," Journal Solid State Chemistry, vol. 79, no. 2, pp. 181-188, 1989.

[28] G. B. Schaffer and P. G. McCormick, "Anomalous combustion effects during mechanical alloying," Metallurgical Transaction A, vol. 22, pp. 3019-3024, 1991.

[29] G. B. Schaffer and J. S. Forrester, "The influence of collision energy and strain accumulation on the kinetics of mechanical alloying," Journal Material Science, vol. 32, pp. 3157-3162, 1997.

[30] L. Takacs and M. A. Susol, "Combustive mechanochemical reactions in off-stoichiometric powder mixtures," Materials Science Forum, vol. 225-227, pp. 559-562, 1996.

[31] L. Takacs, "Combustion phenomena induced by ball milling," Materials Science Forum, vol. 269-272, pp. 513-522, 1998.

[32] F. Charlot, E. Gaffet, B. Zeghmati, F. Bernard, and J. C. Niepce, "Mechanically activated synthesis studied by X-ray diffraction in the Fe-Al system," Material Science Engineering A, vol. 262, no. 1-2, pp. 279-288, 1999.

[33] C. Gras, E. Gaffet, F. Bernard, and J. C. Niepce, "Enhancement of self-sustaining reaction by mechanical activation: case of an Fe-Si system," Material Science Engineering A, vol. 264, no. 12, pp. 94-107, 1999.

[34] C. Deidda, F. Delogu, F. Maglia, U. Anselmi-Tamburini, and G. Cocco, "Mechanical processing and self-sustaining hightemperature synthesis of TiC powders," Material Science Engineering A, vol. 375-377, pp. 800-803, 2004.

[35] C. Deidda, F. Delogu, and G. Cocco, "Changes in the $\mathrm{Ta}_{50} \mathrm{C}_{50}$ mechanochemical reactivity under different milling conditions," Journal Metastable Nanocrystalline Material, vol. 2021, pp. 337-342, 2004. 
[36] C. Caravati, F. Delogu, G. Cocco, and M. Rustici, "Hyperchaotic qualities of the ball motion in a ball milling device," Chaos, vol. 9, no. 1, pp. 219-226, 1999.

[37] G. Manai, F. Delogu, and M. Rustici, "Onset of chaotic dynamics in a ball mill: attractors merging and crisis induced intermittency," Chaos, vol. 12, no. 3, pp. 601-609, 2002.

[38] J. Shäfer, S. Dippel, and D. E. Wolf, "Force schemes in simulations of granular materials," Journal Physique I, vol. 6, no. 1, pp. 5-20, 1996.

[39] G. Manai, F. Delogu, L. Schiffini, and G. Cocco, "Mechanically induced self-propagating combustions: experimental findings and numerical simulation results," Journal Material Science, vol. 39, pp. 5319-5324, 2004.

[40] M. P. Allen and D. Tildesley, Computer Simulations of Liquids, Oxford, Clarendon, TX, USA, 1987.

[41] C. He and G. C. Stangle, "A micromechanistic model of the combustion synthesis process: mechanism of ignition," Journal Material Research, vol. 13, no. 1, pp. 146-155, 1998. 